\title{
Hábitos alimentarios e índice de masa corporal en estudiantes de Medicina de una universidad privada de Lambayeque, Perú, 2019
}

\author{
Juan Antonio Gamero-Baylón 1,a, Juan Daniel Huamanchumo-Benites 1,a, Lucia de Fátima \\ Núñez-Rojas ${ }^{1, a}$, Helen Lizbeth Tello-Huamán ${ }^{1, a}$, Daysi del Carmen Barreto Pérez ${ }^{2, b}$, \\ Antero Enrique Yacarini-Martínez 1,c
}

\section{RESUMEN}

Objetivo. Describir los hábitos alimentarios e índice de masa corporal (IMC) en estudiantes de Medicina de una universidad privada de Lambayeque, año 2019. Materiales y métodos. Estudio descriptivo y transversal, realizado en estudiantes de Medicina de todos los ciclos de la Universidad Santo Toribio de Mogrovejo (USAT). Los hábitos alimentarios se midieron a través dela aplicación de un Cuestionario de Hábitos Alimentarios; mientras que el IMC se midió siguiendo las recomendaciones de la "Guía técnica para la valoración nutricional antropométrica de la persona adulta", del Ministerio de Salud del Perú. La participación de los estudiantes fue a través de firma de un consentimiento informado. Resultados. Se evaluaron a 92 estudiantes entre 17 y 26 años, el 48,9 \% fueron varones. El 29,4\% (27/92) presentaron malos hábitos alimentarios y el 75,0 \% (69/92) no consumía diariamente sus tres comidas. El 35,9 \% (33/92) y el 3,2 \% (3/92) presentaron sobrepeso y obesidad grado 1, respectivamente. Conclusiones. Estudiantes de Medicina de una universidad privada de Lambayeque, Perú, presentaron alta frecuencia de malos hábitos alimentarios y de sobrepeso, por lo que es necesario intervenir con estrategias para prevenir futuras enfermedades nutricionales y metabólicas en esta población.

Palabras Clave: Estudiantes de Medicina, Hábitos alimentarios, Índice de Masa Corporal, Obesidad, Sobrepeso, Perú (Fuente: DeCS-BIREME).

\section{Food habits and body mass index in medical students of a private university, Lambayeque, Perú, 2019}

\section{ABSTRACT}

Objective. Describe the eating habits and body mass index (BMI) in medical students of a private university of Lambayeque, year 2019. Materials and methods. Descriptive and cross-sectional study, carried out in medical students of all cycles of the Universidad Santo Toribio de Mogrovejo (USAT). Eating habits were measured through the application of a Food Habits Questionnaire; while the BMI was measured following the recommendations of the Technical guide for the anthropometric nutritional assessment of the adult, of the Ministerio de Salud del Perú. The participation of the students was through the signing of an informed consent. Results. 92 students between 17 and 26 years old were evaluated, $48.9 \%$ were male. $29.4 \%$ (27/92) had poor eating habits and $75.0 \%$ (69/92) did not consume their three meals daily. $35.9 \%$ (33/92) and 3.2\% (3/92) were overweight and obese grade 1, respectively. Conclusions. Medical students from a private university in Lambayeque, Perú, presented high frequency of poor eating and overweight habits, so it is necessary to intervene with strategies to prevent future nutritional and metabolic diseases in this population.

Keywords: Students Medical, Feeding Behavior; Body Mass Index, Obesity, Overweight, Perú (Fuente: MeSH-NLM).

\footnotetext{
1 Escuela de Medicina Humana, Universidad Católica Santo Toribio de Mogrovejo, Chiclayo, Perú.

2 Servicio de nutrición, Hospital Regional Lambayeque, Chiclayo, Perú.

a Estudiante de Medicina.

${ }^{b}$ Nutricionista.

• Biólogo Microbiólogo. Maestro en Bioética.
} 


\section{INTRODUCCIÓN}

Según la Organización Mundial de la salud (OMS) para el año 2020, unas dos terceras partes de la carga mundial de morbilidad serán atribuibles a enfermedades crónicas no transmisibles. La mayoría de estas enfermedades están relacionadas a hábitos y comportamientos que se aplican día a día en el estilo de vida de las personas. Las conductas alimentarias son una de las principales causas de estas enfermedades, ya que estas condicionarán la calidad dietética y por ende el estado nutricional de las personas, que en el caso de no ser las adecuadas podrían traer consigo patologías como la obesidad, enfermedades cardiovasculares, diabetes mellitus e hipertensión arterial ${ }^{(1,2,3)}$.

En el Perú, el año 2017 la prevalencia de obesidad y sobrepeso en personas de 15 años fue de $18,3 \%$ y 33,8 \%, respectivamente; donde Lambayeque fue la segunda región natural con mayor índice de sobrepeso con 39\%. Estas estadísticas también nos revelan que es dentro de las poblaciones jóvenes donde se encuentra más probabilidades de desarrollar hábitos dietarios que puedan causar defectos nutricionales y desequilibrios, por lo que es en esta población en la cual se tiene que prestar más atención para prevención de las enfermedades crónicas ${ }^{(4,5)}$.

Los cambios bruscos de estilos de vida en los jóvenes es cuando llegan a la etapa de estudios universitarios. La necesidad de comer fuera de casa y sin supervisión de los padres, debido a los compromisos académicos, los vuelve vulnerables a presentar alteraciones alimenticias tras la adopción de comportamientos inadecuados, como los ayunos prolongados o la formación de "patrones dietéticos" en dietas altas en grasas saturadas y azúcares refinados. Los jóvenes universitarios al encontrarse en un estado constante de gasto energético deben suplir estos requerimientos con fuentes o comidas que les cubran estas necesidades, sin embargo, las comidas rápidas y la comida chatarra se encuentran siempre dentro de las opciones al alcance de ellos. La repetición de este comportamiento llevará al desarrollo de hábitos alimentarios inadecuados que pueden originar conductas de riesgo para sufrir enfermedades que afectarán directamente a su salud y desempeño académico ${ }^{(6,7,8)}$. En el Perú, es el Centro Nacional de Alimentación y Nutrición (CENAN), el que atiende este tema de alta trascendencia ya que busca mejorar la situación nutricional y los hábitos alimentarios del poblador peruano mediante la producción de bienes y servicios estratégicos de calidad para contribuir al bienestar de la población ${ }^{(9)}$.

Este problema también afecta, de manera importante, a los estudiantes universitarios, quiénes reciben alta exigencia académica y están sometidos a niveles altos de estrés. Sin embargo, sus hábitos alimenticios y estilo de vida saludables no siempre son los adecuados para sostener físicamente la demanda intelectual a la que son sometidos. Por este mo- tivo, el objetivo de este trabajo es describir los hábitos alimentarios e índice de masa corporal en los estudiantes universitarios de Medicina Universidad Católica Santo Toribio de Mogrovejo (USAT), Lambayeque, Perú, año 2019.

\section{MATERIAL Y MÉTODOS}

\section{Tipo y diseño de investigación}

Estudio observacional descriptivo y transversal.

\section{Población y muestra}

Estudiantes matriculados en el semestre 2019-II de la Escuela de Medicina Humana de la USAT. El tamaño muestral se calculó con el programa OpenEpi con un intervalo de confianza del $95 \%$, un límite de confianza del $5 \%$, y un efecto de diseño de 1,0 . Se obtuvo un tamaño de muestra de 92 estudiantes.

Se utilizó un muestreo por conglomerados, en el que los conglomerados fueron los ciclos pares II, IV, VI, VIII, X y $\mathrm{XII}$, siendo respectivamente los asignaturas de: Introducción a la Medicina, Informática Médica II, Inglés Médico II, Salud Pública III, Especialidades Médicas IV, y Seminario de Tesis III.

\section{Técnicas e instrumentos de recolección de dato}

Se aplicó un cuestionario que se elaboró tomando como base el Cuestionario de hábitos alimentarios (FHQ) realizado originalmente en Estados Unidos, el cual fue adaptado en nuestro país utilizado por Ferro et al. en 320 estudiantes de la Universidad Nacional Mayor de San Marcos $(10,11)$. Dentro del cuestionario donde se recolectaron los datos de peso y talla, siendo las técnicas de recolección de datos de estos últimos dos basados en la R.M. N¹842012/MINSA "Guía técnica para la valoración nutricional antropométrica de la persona adulta". 'Posteriormente se procedió a calcular el índice de masa corporal (IMC) mediante la fórmula (IMC = peso [kg]/ estatura [m2]).

\section{Consideraciones éticas.}

Se aplicó consentimiento informado, a los estudiantes que estén matriculados en ciclo regular y sin ninguna asignatura desaprobada. Así mismo, el estudio fue aprobado por el Comité de Ética en Investigación de la Facultad de Medicina de la USAT.

\section{Análisis de datos}

Se realizó un análisis descriptivo de correspondencia múltiple, utilizando un programa estadístico SPSS 24 y la hoja de cálculo Excel de office 2010 para procesar los datos obtenidos a partir del cuestionario aplicado.

\section{RESULTADOS}

Se evaluaron a 92 estudiantes de pregrado de la Escuela de Medicina Humana de la USAT. La muestra se caracte- 
rizó por varones el $48,9 \%(45 / 92)$ y edades entre 17 y 26 años.

El 60,9\% de los estudiantes presentaron un IMC en el rango normal, no obstante, el $85,7 \%$ de los estudiantes varones del XII ciclo estuvieron dentro de un rango normal; asimismo, el $3,2 \%$ de todos los estudiantes encuestados presentaba obesidad grado 1. El sobrepeso estuvo presente en el 35,9 $\%$ de los estudiantes, la mayor frecuencia estuvo en los estudiantes del X ciclo, donde el 60,0\% eran varones (tabla 1).

En cuanto a los hábitos alimentarios, el 29,4\% tuvieron el calificativo de malo. Asimismo, el $75,0 \%$ de los estudiantes no consumían las tres comidas diarias. Con respecto a los que diariamente consumían sus tres comidas básicas, la cena era la que más comían diariamente con un 75,0 $\%$, seguido del almuerzo con un $63,0 \%$ y del desayuno con un $54,4 \%$. Los estudiantes del II ciclo fueron los que con mayor frecuencia comían diariamente su desayuno, almuerzo y cena con un $73,3 \%, 93,3 \%$ y $86,7 \%$, respectivamente. Por otro lado, dentro de los que nunca consumían cualquiera de sus tres comidas básicas, el almuerzo era el más frecuente con un $15,2 \%$, siendo los del X ciclo los que nunca almorzaba (tabla 1 ).

Tabla 1. Hábitos alimentarios e índice de masa corporal (IMC) en estudiantes de Medicina de la Universidad Santo Toribio de Mogrovejo, Lambayeque, Perú, 2019 (N=92).

\begin{tabular}{lcc}
\multicolumn{1}{c}{ Variable de estudio } & $\mathbf{N}$ & $\%$ \\
Hábitos alimentarios & & \\
Buenos & 65 & 70,6 \\
Malos & 27 & 29,4 \\
Consumo diario de las & & \\
tres comidas & & \\
Sí & 23 & 25,0 \\
No & 69 & 75,0 \\
Índice de masa corporal & & \\
(IMC) & & \\
Normal & 56 & 60,9 \\
Sobrepeso & 33 & 35,9 \\
Obesidad grado 1 & 3 & 3,2 \\
\hline
\end{tabular}

\section{DISCUSIÓN}

Tomando en cuenta las puntuaciones adaptadas del cuestionario de hábitos alimentarios realizado originalmente en Estados Unidos, adaptado por Ferro et al $(10,11)$. Las puntuaciones obtenidas en nuestro estudio salen después de considerar aspectos como frecuencia y horario de comidas, que se consume en cada comida diaria y si es que hay un balance cualitativo de carbohidratos, grasas y proteínas.

Si intentamos relacionar el IMC y los buenos hábitos alimentarios, tomando en cuenta las puntuaciones del cuestionario, encontraríamos una relación por la presencia del $60,9 \%$ de los estudiantes en un rango normal y $70,6 \%$ de los estudiantes poseen hábitos alimentarias adecuados, sin embargo, el porcentaje restante que no coincide se debe probablemente al IMC que no siempre va a depender de los buenos hábitos alimentarios, sino también de la actividad metabólica de cada estudiante, sus condiciones fisiológicas y factores genéticos y epigenéticos.
El estudio nos mostró que únicamente el $25 \%$ de todos los estudiantes encuestados consumían sus tres comidas básicas diariamente, lo cual es un dato de preocupación, ya que solo en los estudiantes de los primeros ciclos se observan porcentajes satisfactorios que indiquen que diariamente consumen sus tres comidas diarias, pero, en los estudiantes de XII ciclo, esto cambia radicalmente, en que ningún estudiante el que almuerce diariamente, a pesar de que a nivel general de todos los ciclos el almuerzo fue el alimento diario más respetado. Los estudiantes de este ciclo propio por los estudios en clínicas y sus cursos más prácticos demuestran que la vida agitada de los estudiantes de medicina se vuelve más intensa cada vez que más se acerca a lograr y ejercer la carrera profesional.

Los resultados de este estudio revelan un problema serio y creciente, por cuanto la exigencia académica e intelectual de los estudiantes de Medicina requiere de un estado nutricional óptimo, que se ve revelado en buenos hábitos alimentarios y un IMC normal. 
Se concluye que el $29,4 \%$ de los estudiantes de Medicina Humana de la USAT presentaron malos hábitos alimentarios y el $60.87 \%$ de los estudiantes evaluados en los diferentes ciclos, tiene un IMC dentro de un rango normal.

Fuentes de financiamiento. La investigación fue autofinanciada.

Conflictos de intereses. Declaro no haber tenido conflictos de intereses.

\section{REFERENCIAS BIBLIOGRÁFICAS}

1. Monge, J. Hábitos alimenticios y su relación con el índice de masa corporal de los internos de enfermería de la U.N.M.S.M. [Tesis en internet, para optar el título profesional de Licenciada en Enfermería]. Lima: Universidad Nacional Mayor de San Marcos, 2007 [citado 20 de enero de 2020]. Disponible en: http://cybertesis.unmsm.edu.pe/bitstream/handle/ cybertesis/478/Monge_dj.pdf?sequence=1.

2. Hernández, C. Comparación de la composición corporal, estilos de vida y consumo de alimentos de un grupo de estudiantes de nutrición y dietética de la Pontificia Universidad Javeriana. Periodo $2005-2008$. [Tesis en internet, para optar el título de Nutricionista Dietista]. Bogotá: Pontificia Universidad Javeriana, 2009 [citado 20 de enero de 2020]. Disponible en: https://repository.javeriana.edu.co/bitstream/handle/10554/8324/ tesis297.pdf?sequence=1\&isAllowed=y.

3. Flegal, K; Carroll, M; Ogden, C; Johnson, C. Obesidad en adultos de Estados Unidos. Rev Panam Salud Pública/Pan Am J Public Health 2002; 12(5).

4. Cervera F, Serrano R, Vico C, Milla M, García M. Hábitos alimentarios y evaluación nutricional en una población universitaria. Nutr Hosp. 2013;28(2):438-46.

5. Ponce G, Ruiz J, Cisneros E, Magaña A, Arizona B. Obesidad y factores de riesgo en estudiantes del área de la salud de la Universidad Autónoma de Baja California, Mexicali. Rev Salud Publica y Nut. 2001;1247):115.

6. Rodríguez F, Palma X, Romo A, Escobar D. Hábitos alimentarios, actividad física y nivel socioeconómico en estudiantes universitarios de Chile. Nutr Hosp. 2013;28 (2):447-55.

7. Ramos JA, Salazar MTL, García G, Hernández MC, Bonilla L. Hábitos de alimentación en estudiantes universitarios [Internet]. II Encuentro Participación de la Maujer en la Ciencia. Puebla. Disponible en: https:// docplayer.es/47742833-Habitos-de-alimentacion-en-estudiantes-universitarios.html.

8. Lameiras M, Calado M, Rodríguez Y, Fernández M. Hábitos alimentarios e imagen corporal en estudiantes universitarios sin trastornos alimentarios. Int J Clin Heal Psychol. 2003; 3(1):23-33.

9. Ministerio de Salud. Conócenos | Instituto Nacional de Salud [Internet]. Disponible en:https://web.ins.gob.pe/es/alimentacion-y-nutricion/acerca-de-cenan/presentacion

10. National cáncer institute. Diet history questionnarie: validation studies [Internet]. USA: NIH; 2002 [Citado el 24 de Agosto de 2018]. Disponible en: https://epi.grants.cancer.gov/DHQ/about/validation.htm.

11. Ferro R, Maguiña V. Relación entre hábitos alimentarios e índice de masa corporal en estudiantes de una universidad pública según área de estudio. [Tesis en internet, para optar el Título Profesional de Licenciada en Nutrición]. Lima: Universidad Nacional Mayor de San Marcos, 2012 [citado 20 de enero de 2020]. Disponible en: http://cybertesis.unmsm. edu.pe/handle/cybertesis/1123.

Revisión de pares: Recibido: 09-12-2019 Aceptado: 21-01-2020 